\title{
Converging Medial Frontal Resting State and Diffusion-Based Abnormalities in Borderline Personality Disorder
}

\author{
Raymond Salvador, Daniel Vega, Juan Carlos Pascual, Josep Marco, \\ Erick Jorge Canales-Rodríguez, Salvatore Aguilar, Maria Anguera, Angel Soto, Joan Ribas, \\ Joaquim Soler, Teresa Maristany, Antoni Rodríguez-Fornells, and Edith Pomarol-Clotet
}

\begin{abstract}
BACKGROUND: The psychological profile of patients with borderline personality disorder (BPD), with impulsivity and emotional dysregulation as core symptoms, has guided the search for abnormalities in specific brain areas such as the hippocampal-amygdala complex and the frontomedial cortex. However, whole-brain imaging studies so far have delivered highly heterogeneous results involving different brain locations.

METHODS: Functional resting-state and diffusion magnetic resonance imaging data were acquired in patients with BPD and in an equal number of matched control subjects ( $n=60$ for resting and $n=43$ for diffusion). While mean diffusivity and fractional anisotropy brain images were generated from diffusion data, amplitude of low-frequency fluctuations and global brain connectivity images were used for the first time to evaluate BPD-related brain abnormalities from resting functional acquisitions.

RESULTS: Whole-brain analyses using a $p=.05$ corrected threshold showed a convergence of alterations in BPD patients in genual and perigenual structures, with frontal white matter fractional anisotropy abnormalities partially encircling areas of increased mean diffusivity and global brain connectivity. Additionally, a cluster of enlarged amplitude of low-frequency fluctuations (high resting activity) was found involving part of the left hippocampus and amygdala. In turn, this cluster showed increased resting functional connectivity with the anterior cingulate.

CONCLUSIONS: With a multimodal approach and without using a priori selected regions, we prove that structural and functional abnormality in BPD involves both temporolimbic and frontomedial structures as well as their connectivity. These structures have been previously related to behavioral and clinical symptoms in patients with BPD.
\end{abstract}

Keywords: ALFF, Amygdala, BPD, Brain connectivity, Diffusion MRI, Resting state

http://dx.doi.org/10.1016/j.biopsych.2014.08.026

Borderline personality disorder (BPD) is a serious and chronic mental disorder characterized by marked impulsivity, emotional instability and interpersonal disturbances. With estimates of $5.9 \%$ prevalence in the general population, BPD is the most common personality disorder (1). It is considered to be a difficult-to-treat disorder, with severe functional impairment, high costs to society, and associations with high rates of comorbidity and suicide. Although BPD has been related to the occurrence of childhood negative events (2), it has fairly high heritability levels (65\%-75\%) as reported in twin studies (3).

The psychological profile of BPD, with disinhibition and emotional dysregulation as core symptoms, suggests the possible impairment of frontal and limbic structures and of their connections (4). This suggestion has prompted the search for abnormalities in specific brain locations, such as the hippocampal-amygdala complex, potentially involved in the prevalent negative emotions observed in the disorder. Volumetric studies of this complex, summarized in a meta-analysis
(5), have shown consistent bilateral reductions in both amygdala and hippocampus. However, such results have not been replicated in the only two studies $(6,7)$ that have carried out whole-brain unbiased (in terms of not favoring preselected areas) morphometric comparisons.

The medial frontal cortex, implicated in cognitive control and regulation of emotions (8), has also been a target of BPD studies, and although it has not been reported to be significant in whole-brain morphometric comparisons, it has been found to be abnormal in studies of function (9-12). The medial frontal cortex has extensive connections with the medial temporal cortex (including the amygdala and hippocampus) ensuring the cognitive-emotional control of behavior (13). It has also been shown that the experimental disruption of these connections leads to behavioral patterns similar to those observed in individuals with BPD (14). This evidence has led to the performance of functional magnetic resonance imaging (MRI) studies of connectivity in BPD, which also have reported 
significant alterations $(15,16)$. However, similar to volumetric studies, the scope of such connectivity results is restricted by the prior selection of brain areas of interest. In that sense, the discovery of intrinsic brain networks in the resting brain (17) has allowed analyses with a much broader extent showing various intranetwork and internetwork abnormalities $(18,19)$. Still, such results are relevant only to the specific networks analyzed.

This limitation in the scope of connectivity results has been overcome more recently with a set of methods that are able to summarize the connectivity levels at each location of the brain, delivering individual maps of global brain connectivity (GBC). Voxels in these maps may contain the average correlation with the remaining gray matter voxels (20), the number of connections (edges) reaching the voxel defined by a binary matrix $(21,22)$ or values of global covariability in the frequency domain $(23,24)$. None of these methods have been applied so far in patients with BPD.

Functional connectivity findings may be compared with findings from structural or anatomic connectivity (derived from diffusion MRI). However, apart from weak evidence of orbitofrontal white matter deterioration in BPD (25-27), results with this modality are still scarce. Alternatively, resting-state functional MRI may be used to quantify the amplitude of blood oxygen level-dependent fluctuations, which are usually restricted to the low frequencies (e.g., .01-.1 Hz) and are commonly known as analyses of amplitude of low-frequency fluctuations (ALFF) (28). These values, which are taken as indicators of spontaneous brain activity, have been linked to physiologic states (29), to levels of cerebral blood flow (30), and to local field potentials (31). Although functional connectivity analyses such as the GBC quantify the levels of covariability between areas of the brain, ALFF analyses are concerned only with reporting the net activity levels at each brain site, regardless of the activity in other brain areas (i.e., ALFF is not a type of connectivity analysis). So far, no study of ALFF in BPD has been published.

In the present study, employing a large sample of patients with BPD ( $n=60$ for resting functional MRI and $n=43$ for diffusion MRI) matched to equally sized samples of healthy control subjects, we performed a whole-brain multimodal MRI analysis of the disorder. Specifically, resting functional MRI is used to 1) calculate GBC maps, in which each voxel contains the mean correlation with each of the remaining gray mater voxels, and 2) estimate images of ALFF; this is combined with brain maps of mean diffusivity (MD) and fractional anisotropy (FA) derived from diffusion MRI images, which are compared between patients with BPD and healthy control subjects. We hypothesize that the joint analysis of all these measures would give us a coherent picture of the abnormal brain connectivity patterns underlying this disorder. More specifically, we expect to confirm frontolimbic abnormality without the need to set a priori anatomic restrictions in the analyses.

\section{METHODS AND MATERIALS}

\section{Sample}

An original sample of 76 female outpatients with a diagnosis of BPD was recruited from two Spanish BPD specialist units located in Barcelona (Hospital de la Santa Creu i Sant Pau) and Igualada (Consorci Sanitari de l'Anoia). The main reason for selecting an all-female group was the high prevalence of women $(\sim 90 \%)$ in the clinical samples attending both recruiting units. Although a small subsample of men was available, it was not included to avoid the potential effect of intergender variability in the MRI images (32).

The diagnosis of BPD was established with the validated Spanish versions of two semistructured questionnaires: the Structured Clinical Interview for DSM-IV Axis II disorders (33), which assesses personality disorders according to DSM-IV and has shown an adequate interrater reliability and discrimination among Axis II personality disorders, and the Revised Diagnostic Interview for Borderlines (DIB-R) (34), an instrument that provides the diagnosis of BPD based on information from the last 2 years. In addition to these diagnostic questionnaires, the Borderline Symptom List-23 (BSL-23) (35), a 23-item self-rating instrument used to assess the level of clinical severity in BPD, was also applied to a subsample of the patients. Specifically, we used a validated Spanish version with good psychometric properties (36).

Selected patients were right-handed and 18-55 years old. Exclusion criteria included history of brain trauma, history of neurological disease, presence of alcohol or substance abuse or dependence in the previous 6 months, current comorbid Axis I disorder (according to DSM-IV), and previous bipolar or psychotic diagnosis (according to DSM-IV). Patients were allowed to take pharmacologic treatment.

Only images that passed a set of quality control measures described subsequently were kept for the study. These included images of 60 patients for resting-state functional $\mathrm{MRI}$ and of 43 patients for diffusion MRI. Following the same general inclusion criteria, equally sized samples of control subjects (women) were recruited from nonmedical staff working in the hospitals, their relatives and acquaintances, and independent sources in the community. Potential control subjects who reported a history of mental illness or treatment with psychotropic medication were rejected. Healthy women were recruited matching the sample of patients for age and premorbid IQ, which was estimated from two of the subtests (Vocabulary and Matrix) of the Wechsler Adult Intelligence Scale-III. All participants gave written informed consent, and the study was approved by the research ethics committee of both recruiting institutions. Demographic and clinical traits for the final samples are summarized in Table 1.

\section{Magnetic Resonance Imaging Data Acquisition and Processing}

All subjects were scanned with a 1.5-tesla GE Signa scanner (General Electric Medical Systems, Milwaukee, Wisconsin) located in the Sant Joan de Déu Hospital in Barcelona applying parameters used in two previous studies $(24,37)$ and listed in Supplement 1. After several preprocessing steps on the diffusion images (see Supplement 1), the dtifit function implemented in the FMRIB Software Library (38) was used to obtain the images of MD and FA for each subject (Figure 1). While the analysis of MD included all brain parenchyma (both gray and white matter tissues), the analysis of FA abnormalities was restricted to the white matter tracts, characterized by the tract-based spatial statistics procedure included in the 
Table 1. Main Demographic and Clinical Characteristics of Patients and Control Subjects Analyzed with the Two Magnetic Resonance Imaging Modalities

\begin{tabular}{|c|c|c|c|}
\hline \multicolumn{4}{|c|}{ Resting State Functional MRI } \\
\hline & Patients $(n=60)$ & Control Subjects $(n=60)$ & $p$ Value \\
\hline Age (Years) & $32.12(7.16)$ & $33.73(12.8)$ & .398 \\
\hline Gender & All female & All female & NS \\
\hline Estimated Premorbid IQ ${ }^{a}$ & $96.7(14.8)$ & $98.5(10.1)$ & .466 \\
\hline DIB-R Total & $7.76(1.20)$ & NA & \\
\hline BSL-23 ${ }^{b}$ & $56.37(25.84)$ & NA & \\
\hline \multicolumn{4}{|l|}{ Diffusion MRI } \\
\hline & Patients $(n=43)$ & Control Subjects $(n=43)$ & $p$ Value \\
\hline Age (Years) & $31.55(7.32)$ & $32.40(11.8)$ & .694 \\
\hline Gender & All female & All female & NS \\
\hline Estimated Premorbid $\mathrm{IQ}^{a}$ & $94.67(12.5)$ & $95.88(9.74)$ & .619 \\
\hline DIB-R Total & $7.72(1.16)$ & NA & \\
\hline $\mathrm{BSL}-23^{\mathrm{C}}$ & $55.72(23.93)$ & NA & \\
\hline
\end{tabular}

Complementing mean values, standard deviations are provided inside parentheses. BSL-23, Borderline Symptom List-23; DIB-R, Revised Diagnostic Interview for Borderlines; MRI, magnetic resonance imaging; NS, not significant.

${ }^{a}[($ Wechsler Adult Intelligence Scale-III Vocabulary) + (Wechsler Adult Intelligence Scale-III Matrix)] × 11/2.

${ }^{b} n=43$ (available subsamples).

${ }^{c} n=32$ (available subsamples).

FMRIB Software Library (FMRIB Software Library-Tract-Based Spatial Statistics) (39) (see more details in Supplement 1).

Resting-state images were used to calculate both GBC maps and the images of ALFF. Before their calculation, a common preprocessing pipeline was applied to them (Supplement 1). In the GBC calculations, following Cole et al. (20), the correlation between each gray matter voxel and the remaining gray matter voxels was calculated, assign-
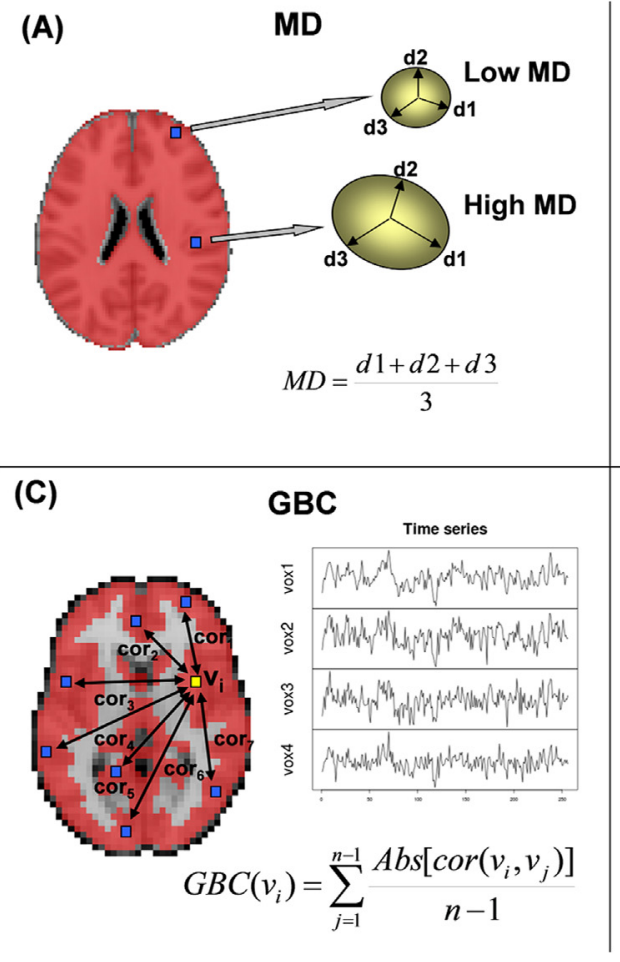

(B)

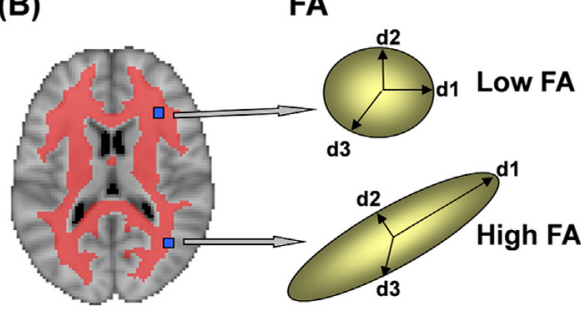

$$
F A=\sqrt{\frac{3}{2}(d 1-M D)^{2}+(d 2-M D)^{2}+(d 3-M D)^{2}} \frac{d 1^{2}+d 2^{2}+d 3^{2}}{d}
$$

(D)

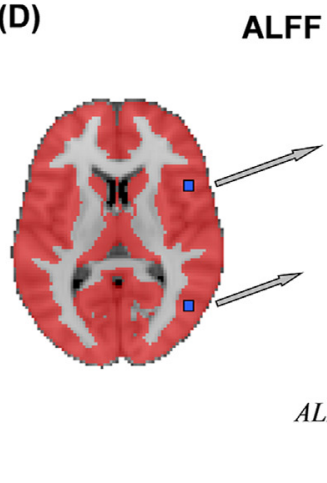

FA

F

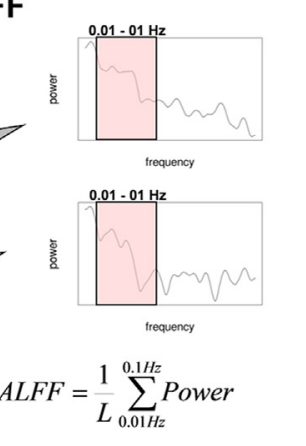

Figure 1. Simple visual explanation of the four magnetic resonance imaging quantities evaluated in this study. While mean diffusivity and fractional anisotropy are extracted from diffusion images, global brain connectivity and amplitude of lowfrequency fluctuations are calculated from resting-state functional images. (A) Mean diffusivity measures the average diffusion of water in the three orthogonal axes of maximum diffusion in the voxels of the brain parenchyma. (B) Fractional anisotropy quantifies the amount of directionality of the diffusion (eccentricity of the diffusion ellipsoid) in the white matter. (C) The global brain connectivity at each voxel is characterized here by the mean of the absolute values of its correlations with the remaining voxels of the gray matter, using a lower resolution $\left(4 \mathrm{~mm}^{3} \times 4 \mathrm{~mm}^{3} \times 4 \mathrm{~mm}^{3}\right.$ voxel size). This quantity is an indicator of the average levels of functional connectivity of the voxel with the rest of the brain. (D) The amplitude of lowfrequency fluctuations of each gray matter voxel is obtained by averaging its estimate of the power spectrum in the .1-.01 Hz interval, and is used as an indicator of the spontaneous levels of brain activity of the voxel. ALFF, amplitude of low-frequency fluctuations; FA, fractional anisotropy; GBC, global brain connectivity; $L$, length quantified by the number of Fourier coefficients in the interval; MD, mean diffusivity. 
ing the mean (of the absolute value) of these correlations to that voxel. Such quantity is an indicator of the average levels of functional connectivity of the voxel with the rest of the brain (Figure 1C). For the ALFF images, which relate to the levels of spontaneous brain activity occurring at each voxel, periodograms (estimates of power spectra) were obtained using the function spec.pgram implemented in the $R$ statistical package (40), and averages of these periodograms in the $.01-0.1 \mathrm{~Hz}$ interval were given as outputs (Figure 1D). See Supplement 1 for a full description of GBC and ALFF calculations.

\section{Group Comparisons}

For the four variables of interest (MD, FA, GBC, and ALFF), images of the patient sample were compared with images of controls by means of nonparametric permutation tests. Specifically, the "randomise" FMRIB Software Library function (38) using the threshold-free cluster enhancement method was used. To reduce the intrinsic levels of variability and the residual movement effects in the GBC and ALFF images, the average amount of movement and the average value of each variable were considered as covariates in the models. A threshold of $p<.05$ corrected for multiple comparisons was applied in all statistical tests, and reported $p$ values were always corrected, with the exception of one exploratory cluster shown in Figure 3.

\section{Relation with Clinical Condition}

To explore the potential relationship between imaging findings and clinical traits, the intensity of brain abnormalities found in the group analyses was correlated with several clinical indexes. Specifically, in the sample of patients, mean values of voxels with significant increases or decreases in MD, FA, ALFF, and GBC were correlated with 1) the general measure of clinical severity provided by the BSL-23; 2) emotion regulation as registered in the DIB-R; and 3) impulsivity, also as recorded within the DIB-R.

\section{Evaluation of Cross-Modal Convergence}

Finally, examination of convergence of abnormalities across modalities was carried out through overlays of images including significant clusters from the different modalities. While logical intersections were used to detect "tight convergence" (anatomic overlap between modalities), images of logical unions (including nonoverlapping clusters) were visually inspected to describe, in an informal way, brain anatomic regions that contained anomalies from different modalities.

\section{RESULTS}

\section{Diffusion}

The comparison of MD images only showed increases in patients with BPD relative to control subjects. These increases were observed in 11 clusters, mainly located in the anterior part of the brain, in areas with a considerable fraction of gray matter (first row of Figure 2 and Table 2). These spanned bilaterally from lateral orbitofrontal structures to both insulae and up to precentral and postcentral cortices, also reaching the anterior part of the temporal lobes. In addition, a single cluster of increased MD was located medially in the anterior cingulate.

The analysis of FA, which was carried out in white matter, only showed BPD-related reductions. These occurred in tracts located frontally, with most extended alterations in the genu and body of the corpus callosum but also involving part of the corona radiata, external capsule (including uncinate fasciculus and inferior fronto-occipital fasciculus), and other white matter structures (second row of Figure 2 and Table 2).

\section{Functional Resting State}

Both BPD-related increases and reductions in functional connectivity were shown in GBC comparisons. While abnormally high GBC values were observed in a single cluster located in the pregenual anterior cingulate, reduced GBC in patients with BPD was found only in the right temporal lobe (third row of Figure 2 and Table 2). Likewise, ALFF analyses also showed both significant increases and reductions in amplitudes. Increases were observed in a cluster partially involving the left hippocampus and amygdala and in another cluster located in the left putamen. Reductions were located posteriorly in the occipital lobes, in the right precuneus, and in the dorsalposterior cingulate cortex (last row of Figure 2 and Table 2).

\section{Relation to Clinical Condition}

Results of correlations between levels of abnormality in MD, FA, ALFF, GBC and clinical severity scores from the BSL-23, emotion regulation and impulsivity (both from DIB-R) are shown in Table 3. For the BSL-23, only the mean ALFF for areas with increased amplitude in patients showed a moderate, but significant, correlation with this measure of global clinical severity. This correlation was in the expected direction (i.e., more ALFF, more severity). Results for MD, although at trend level $(p<.1)$, also point to a possible positive relationship with clinical severity.

For the DIB-R, no imaging result was linked to impulsivity, whereas only the area of reduced GBC in patients was significantly correlated with emotion regulation. As expected, this relationship was negative (more dysregulation linked to lower GBC). All significant correlations reported here should be taken as exploratory because they were moderate in magnitude and did not stand a correction for multiple comparisons.

\section{Convergence of Modalities and Follow-Up Seed Analyses}

A combined view from different modalities shows evidence of an anatomic convergence of results in genual and perigenual areas (a location frequently reported in previous BPD studies). As shown in Figure 3, white matter FA abnormalities partially encircled clusters significant in MD and GBC comparisons. In contrast, the hippocampus-amygdala complex (also the focus of many previous BPD studies) was significant only in the ALFF analyses. To explore potential abnormalities in this complex further, we took the significant cluster in the left hippocampusamygdala as a seed for a functional connectivity analysis, and this seed showed only abnormal (high) correlations with a small 
cluster also located in the anterior cingulate (Figure 3). Finally, we also performed a follow-up study taking the two significant clusters from the GBC analysis as seeds for a functional connectivity analysis (Figure S1 in Supplement 1).

\section{DISCUSSION}

In the present study, we combine resting-state and diffusionbased brain maps to characterize abnormalities in patients with BPD. Because the analyses we performed take a wholebrain approach, not considering a priori regions, results are not anatomically constricted as in some preceding studies. For the first time, we also implement two resting-state measures (ALFF and GBC) to evaluate BPD.

Although differences are present in various areas of the brain, of special interest are the multimodal abnormalities observed in frontomedial structures, which match previous findings in BPD and, as stated at the beginning of this article, may explain many of the behavioral traits of the disorder. The specific perigenual location of the abnormalities overlaps with areas shown to be actively involved in cognitive control, impulsivity, reward, and modulation of emotions $(8,41)$, all of which are altered in BPD.
From the structural point of view, reported white matter frontal abnormalities agree with some of the previous studies (25-27), but the observed bilateral increases in MD, mainly in gray matter, are new. Changes in MD have been previously related to gray matter changes (42) suggesting that the increases in MD in our study are probably linked to reduction in gray matter volumes in the same areas. However, there is only one voxel-based morphometric study on BPD reporting similar extensive bilateral alterations (43), and it used a lenient uncorrected $p$ value of .001 .

Among other frontal white matter structures, decreases in FA were observed in the uncinate and in the inferior frontooccipital fasciculi of the corona radiata. Similar reductions have been observed in patients with psychopathy, which were interpreted in terms of a possible decrease in connectivity in the amygdala-orbitofrontal limbic network through the uncinate fasciculus (44). Another study has also showed reductions of FA in this region associated with reward and punishment susceptibility (45), two aspects that are altered in patients with BPD (46), while Joutsa et al. (47) reduced white matter integrity in both fasciculi was observed in pathological gamblers. The uncinate fasciculus is a ventral associative pathway that connects the anterior temporal lobe with the
MEAN DIFFUSIVITY (MD)

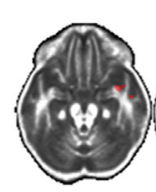

$Z=-20$ $Z=-15$

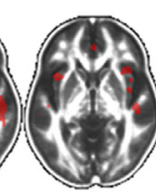

$Z=-3$

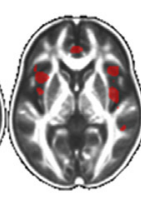

$Z=2$

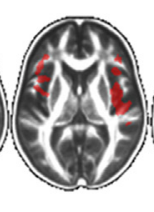

$Z=6$

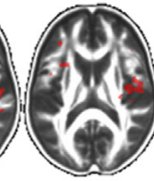

$Z=12$

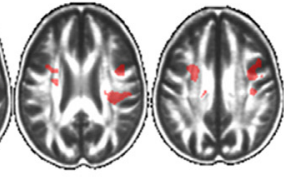

$Z=22$

$Z=32$

FRACTIONAL ANISOTROPY (FA)
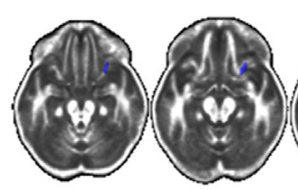

$Z=-15$
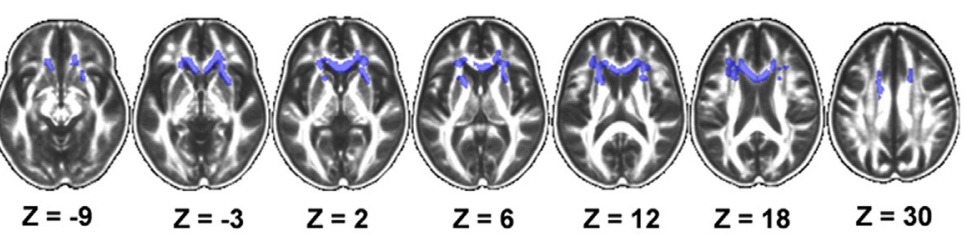

\section{GLOBAL BRAIN CONNECTIVITY (GBC)}

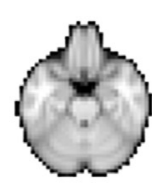

$Z=-24$

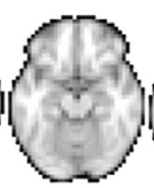

$Z=-16$

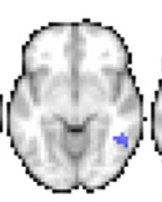

$z=-8$

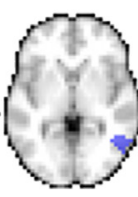

$\mathrm{Z}=0$

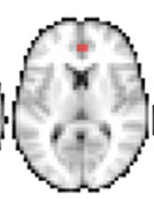

$z=8$

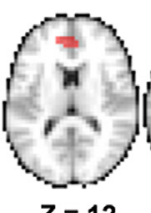

$z=12$

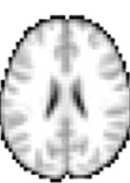

$\mathrm{Z}=\mathbf{2 4}$

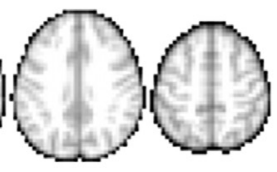

$\mathrm{Z}=36$

$Z=52$
Figure 2. Group differences between patients with borderline personality disorder and control subjects for the four variables analyzed. Significant increases in borderline personality disorder are shown in red, and significant reductions are shown in blue (all results are based on a $p_{\text {corrected }}<.05$ threshold). Increases in mean diffusivity are spread in anterior and medial regions, whereas fractional anisotropy shows reductions only in frontal white matter. An increase in global brain connectivity related to borderline personality disorder was found in the anterior cingulate, and a decrease occurred in the right temporal region. Finally, increases in amplitude of low-frequency fluctuations were found in left subcortical and limbic structures (including the amygdala), and reductions were observed in occipital and medial parietal regions. Left side of the image is left side of the brain. Z Montreal Neurological Institute coordinates for the axial slices are shown for each modality.
AMPLITUDE OF LOW FREQUENCY FLUCTUATIONS (ALFF)
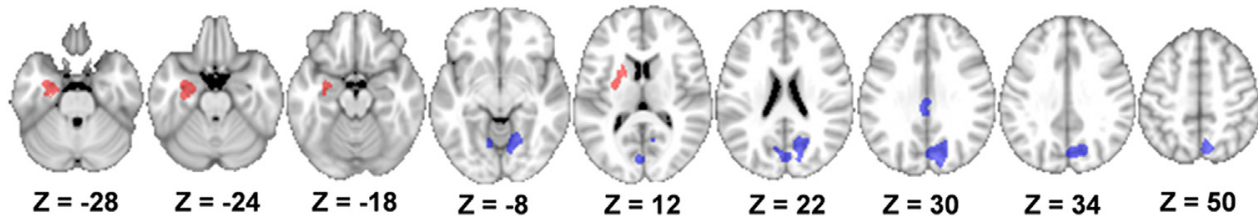

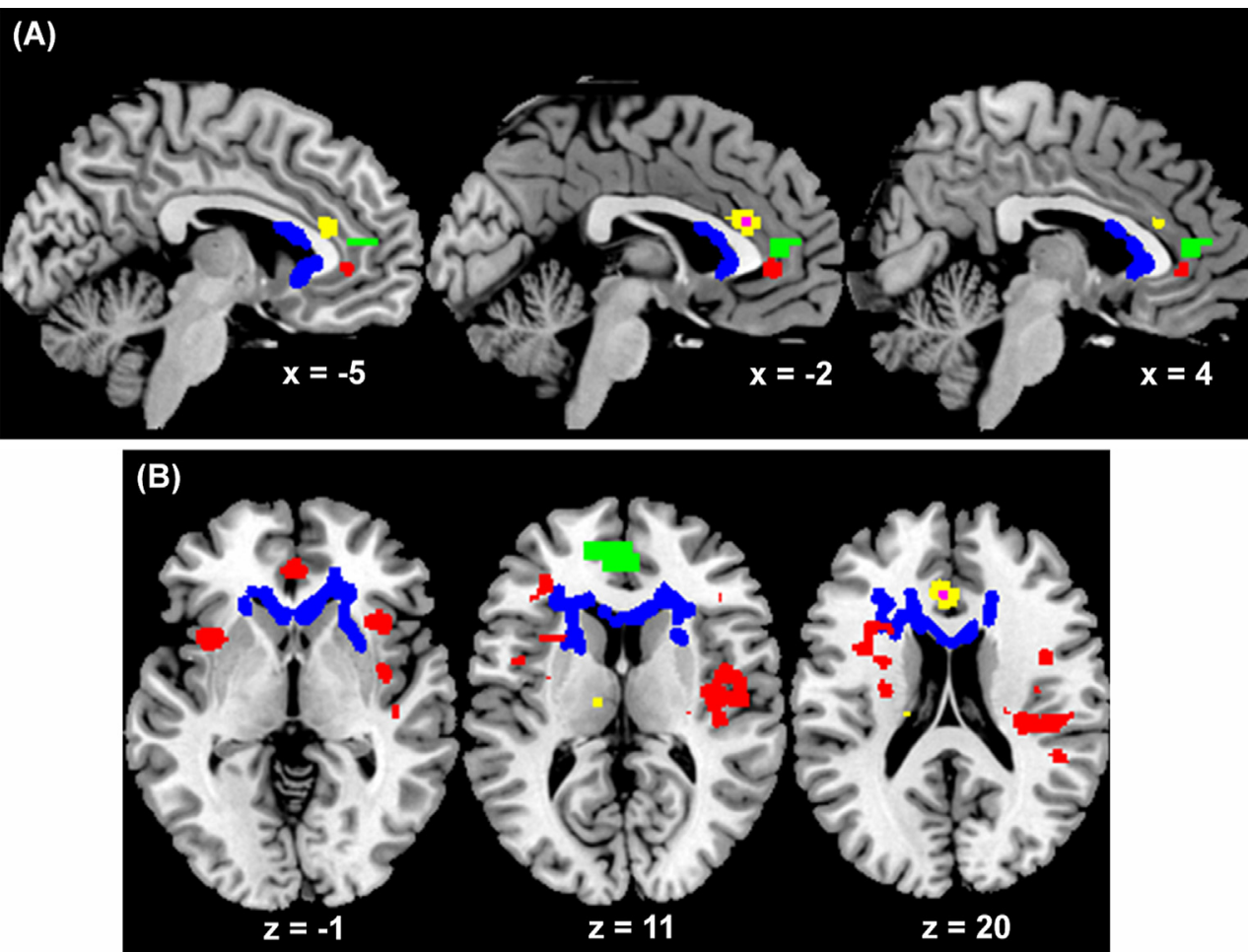

Figure 3. Converging genual and perigenual abnormalities in borderline personality disorder. An overlap of several contrasts involving medial-frontal abnormalities are shown in sagittal (A) and axial (B) slices. White matter alterations in fractional anisotropy shown in blue enclose medial clusters of: 1) altered mean diffusivity shown in red, 2) abnormal global brain connectivity shown in green, and 3) a small region of increased connectivity with the left amygdala (cluster of abnormal amplitude of lowfrequency fluctuations), which is shown in purple $\left(p_{\text {corrected }}<.05\right)$ with an enlarged version ( $p_{\text {uncorrected }}$ $<$.001) shown in yellow.

Table 2. Significant Clusters Found in the Comparison Between Patients with Borderline Personality Disorder and Control Subjects for the Four Parameters Analyzed

\section{Significant Clusters}

MD $\left(1.5 \mathrm{~mm}^{3}\right.$ Final Voxel Size)

BPD patients > control subjects Left insula and nearby cortex, left dorsal cingulate, left pre- and postcentral cortex; 3478 voxels; peak at $\mathrm{MNI}-25.5,4.5,33(p=.0154)$

Right insula and nearby cortex, right medial and superior temporal cortex, part of right amygdala and hippocampus; 3276 voxels; peak at $\mathrm{MNI} 46.5,-13.5,-16.5(p=.0158)$

Right pre- and postcentral cortex; 791 voxels; peak at MNI 43.5, $0,22.5(p=.0254)$

Right insula, right frontal inferior cortex; 341 voxels; peak at MNI $37.5,18,0$ ( $p=.031)$

Left middle and superior temporal cortex; 277 voxels; peak at MNI $-49.5,-33,-9(p=.0414)$

Bilateral anterior cingulate; 174 voxels; peak at MNI 1.0, 40.5, 0 ( $p=.0394)$

Other smaller clusters mainly in right temporal cortex

Control subjects $>$ BPD patients None

FA $\left(1.0 \mathrm{~mm}^{3}\right.$ Final Voxel Size $)$

BPD patients $>$ control subjects None

Control subjects > BPD patients Body and genu of corpus callosum; bilateral anterior corona radiata; 2635 voxels; peak at MNI $-15,16,23(p=0328)$ Right external capsule (mainly uncinate fasciculus and inferior fronto-occipital fasciculus); 361 voxels; peak at MNI 24 , $15,-6(p=.0438)$

Left anterior limb of internal capsule; 312 voxels; peak at MNI -22, 12, $9(p=.0439)$

GBC $\left(4.0 \mathrm{~mm}^{3}\right.$ Final Voxel Size $)$

BPD patients $>$ control subjects Bilateral anterior cingulate; 21 voxels; peak at MNI $-2,46,12(p=.0482)$

Control subjects > BPD patients Right medial and inferior temporal cortex; 53 voxels; peak at MNI $50,-54,-4(p=.036)$

ALFF $\left(2.0 \mathrm{~mm}^{3}\right.$ Final Voxel Size $)$

BPD patients $>$ control subjects Left amygdala-hippocampus; 306 voxels; peak at MNI $-26,-8,-24(p=.0176)$

Left putamen; 370 voxels; peak at MNI $-20,4,10(p=.0144)$

Control subjects > BPD patients Right precuneus and lingual, left and right cuneus and calcarine; other occipital regions, part of right cerebellum; 1662 voxels; peak at MNI 6, $-72,36(p=.0078)$

Left and right dorsal-posterior cingulate; 172 voxels; peak at MNI 0, $-24,26(p=.0226)$

Left lingual and cerebellum; 125 voxels; peak at $\mathrm{MNI}-10,-62,-12(p=.0348)$

Results reported always used a $p_{\text {corrected }}<.05$ for multiple comparisons.

ALFF, amplitude of low-frequency fluctuations; BPD, borderline personality disorder; FA, fractional anisotropy; GBC, global brain connectivity; $\mathrm{MD}$, mean diffusivity; MNI, Montreal Neurological Institute. 
Table 3. Correlations Between Mean Values of Significant Voxels from the Four Imaging Analyses and Scores from the BSL 23, Emotion Regulation and Impulsivity in Patients

\begin{tabular}{|c|c|c|c|}
\hline & BSL-23 & $\begin{array}{l}\text { Emotion Regulation } \\
\text { (DIB-R) }\end{array}$ & $\begin{array}{c}\text { Impulsivity } \\
\text { (DIB-R) }\end{array}$ \\
\hline \multicolumn{4}{|l|}{ MD } \\
\hline $\begin{array}{l}\text { BPD patients > } \\
\text { control subjects }\end{array}$ & $\begin{aligned} r & =.308 \\
p & =.0861^{a} \\
n & =32\end{aligned}$ & $\begin{aligned} r & =-.099 \\
p & =.526 \\
n & =43\end{aligned}$ & $\begin{array}{l}r=.148 \\
p=.342 \\
n=43\end{array}$ \\
\hline \multicolumn{4}{|l|}{ FA } \\
\hline $\begin{array}{c}\text { Control subjects }> \\
\text { BPD patients }\end{array}$ & $\begin{aligned} r & =.047 \\
p & =.799 \\
n & =32\end{aligned}$ & $\begin{array}{l}r=-.152 \\
p=.329 \\
n=43\end{array}$ & $\begin{array}{l}r=-.095 \\
p=.543 \\
n=43\end{array}$ \\
\hline \multicolumn{4}{|l|}{ GBC } \\
\hline $\begin{array}{l}\text { BPD patients > } \\
\text { control subjects }\end{array}$ & $\begin{array}{l}r=-.028 \\
p=.858 \\
n=43\end{array}$ & $\begin{array}{l}r=.143 \\
p=.307 \\
n=60\end{array}$ & $\begin{array}{l}r=.017 \\
p=.905 \\
n=60\end{array}$ \\
\hline $\begin{array}{c}\text { Control subjects }> \\
\text { BPD patients }\end{array}$ & $\begin{array}{l}r=.037 \\
p=.810 \\
n=43\end{array}$ & $\begin{aligned} r & =-.287 \\
p & =.037^{b} \\
n & =60\end{aligned}$ & $\begin{aligned} r & =.029 \\
p & =.839 \\
n & =60\end{aligned}$ \\
\hline \multicolumn{4}{|l|}{ ALFF } \\
\hline $\begin{array}{l}\text { BPD patients > } \\
\text { control subjects }\end{array}$ & $\begin{aligned} r & =.343 \\
p & =.024^{b} \\
n & =43\end{aligned}$ & $\begin{array}{l}r=.032 \\
p=.817 \\
n=60\end{array}$ & $\begin{array}{l}r=-0.119 \\
p=.396 \\
n=60\end{array}$ \\
\hline $\begin{array}{c}\text { Control subjects > } \\
\text { BPD patients }\end{array}$ & $\begin{array}{l}r=-.083 \\
p=.595 \\
n=43\end{array}$ & $\begin{aligned} r & =-.047 \\
p & =.735 \\
n & =60\end{aligned}$ & $\begin{array}{l}r=.204 \\
p=.142 \\
n=60\end{array}$ \\
\hline
\end{tabular}

ALFF, amplitude of low-frequency fluctuations; BPD, borderline personality disorder; BSL-23, Borderline Symptom List-23; DIB-R, Revised Diagnostic Interview for Borderlines; FA, fractional anisotropy; GBC, global brain connectivity; MD, mean diffusivity; MNI, Montreal Neurological Institute.

${ }^{a} p<.1$.

medial and lateral orbitofrontal cortex $(48,49)$ being implicated in emotion and memory processing $(50,51)$. Considering the converging evidence across all these studies, such tracts seem to be important for the regulation of impulsive and reward-related behaviors, as would be the case in patients with BPD.

We also found a reduction in white matter in the genu and body of the corpus callosum. This result suggests a possible alteration in interhemispheric structural connectivity in prefrontal regions, which is in congruence with several previous studies in BPD $(27,52)$ (but also see Zanetti et al. (53) for contradictory evidence). Fibers connecting orbitofrontal and ventral medial prefrontal cortex pass through the genu of the corpus callosum (54). Disrupted connectivity in the corpus callosum has also been observed in association with affective dysregulation in bipolar disorder $(55,56)$, which has a close symptomatic overlap with BPD. Several studies have reported a possible relationship between childhood abuse, trauma, and reduced size of this structure $(57,58)$, including the study by Rusch et al. (52) carried out on patients with BPD. Further research using tractographic tools will eventually be required to characterize the scope and effects of corpus callosum abnormalities on interhemispheric connectivity.

The hippocampal-amygdala complex, a frequent target in earlier studies using regions of interest, has been shown to be significant in the whole-brain analysis of ALFF. The abnormality observed in the left hippocampus and amygdala involves increased resting-state fluctuations in patients with BPD, which may be interpreted as increased activity of these structures at rest. At first glance, this finding may seem to conflict with the meta-analysis by Ruocco et al. (12) reporting less activation in the amygdala under negative stimuli, which may be taken as an indication of the amygdala underreacting in patients with BPD. However, because task-based functional MRI studies are bound to compare brain activity in relation to a baseline, we may conclude from our results that such underactivation is probably due to the fact that the amygdala is already overactive at rest. Such hyperactivity of the amygdala is likely to be part of the neurobiological substrate for the clinical emotional hyperreactivity typical of BPD.

In contrast, BPD-related reductions in ALFF occur far from frontal and limbic regions. Although these results cannot be easily related to previous BPD findings, a similar pattern of ALFF reductions has been reported in schizophrenia $(59,60)$, depression (61), and obsessive-compulsive disorder (62), and it is probably associated with nonspecific deficits occurring in all these disorders.

The increase in GBC observed in the anterior cingulate is relevant. It also agrees with the ancillary analysis showing increased connectivity between the left hippocampus and amygdala cluster (derived by ALFF) and a single small frontomedial cluster. All of these findings agree with the study by Cullen et al. (15), which observed increased connectivity between a priori selected amygdala regions of interest and perigenual cortex under overt fear stimuli. Increase in GBC in the anterior cingulate is also relevant because this area is a core node of the default mode network (63). This network is active during resting periods and is associated with selfreference evaluations, inward attention, and autobiographical memory retrieval $(64,65)$. Since patients with BPD experience constant conflict owing to emotional dysregulation problems, the observed increase in anterior cingulate connectivity may be related to an overuse caused by constant internal thinking and processing of internal conflict.

The observed left amygdala-anterior cingulate hyperconnectivity is also in agreement with the role assigned by theoretical and animal studies to frontolimbic connections as modulators of cognitive-emotional control of behavior $(13,14)$ and could also be the cause of the interferences reported in functional MRI studies combining emotional and cognitive or inhibition tasks (66-68). Overall, our results support the theoretical model in which the amygdala, associated with emotional processing, is modulated by frontomedial structures controlling the complex manifestations and behavior derived from these same emotions. In BPD, alterations of such modulation (connectivity) would act as inducers of some of its clinical traits.

Correlations found between levels of imaging abnormality and registered clinical features give some further support for the consistency of reported brain alterations. Higher levels of clinical severity (BSL-23 scores) seem to be linked to both abnormally increased fluctuations (greater ALFF) and larger MD values in patients. Nevertheless, these relationships should be taken as soft evidence because correlations are moderate and do not survive correction for multiple comparisons. On the other hand, although a significant and interesting relationship is observed between abnormally reduced levels of 
GBC and emotional dysregulation, the remaining comparisons based on the DIB-R questionnaire are not significant. This may be due to the fact that, the DIB- $R$ is mainly a diagnostic tool and not a rating scale designed for quantifying clinical severity.

Finally, results from follow-up connectivity studies based on the two significant GBC clusters (Figure S1 in Supplement 1) also warrant comment. First, the lack of significant connectivity abnormalities in the pregenual cluster (of increased GBC in patients) may be seen as unexpected. Because the GBC is an average of correlations with all gray matter voxels, the most plausible explanation for such a result is that the increase in pregenual GBC is induced by a moderate but consistent gain in connectivity with a large set of different brain regions, none of them individually reaching statistical significance. This set would also include the GBC cluster in the right temporal lobe, for which the seed analysis shows significantly larger connectivity with the medial frontal cortex. However, because the cluster in the right temporal lobe has reduced GBC in patients, that result may look odd, having a plausible explanation only in a moderate but generalized reduction in correlations with a large set of other brain regions.

Our study has some methodologic limitations. First, patients with BPD were taking medication, something that could have potentially modified the results. Patients (and control subjects) recruited were all women, and in a strict sense, conclusions apply only to this gender. In addition, MRI sequences used had some constraints; most notably, functional MRI images were acquired with a wide slice thickness of $7 \mathrm{~mm}$ (plus an interslice gap of .7 mm), which was not optimal for detecting changes in small brain structures. Also, although not a limitation, mean individual values were entered as covariates in the ALFF and GBC comparisons to reduce variability, and consequently results reported for these parameters refer to differences from each subject mean.

The usage of corrected $p$ values throughout the analyses and the above-mentioned whole-brain unbiased approach are strengths of this work. We are aware that such an approach is complementary to seed-based analyses, which, appropriately focused on specific regions known to be related to BPD symptoms, may convey very relevant information for the disorder, otherwise overlooked by a whole-brain approach. Lastly, the inclusion of other clinical aspects, such as the potential role of previous trauma events on the imaging modalities analyzed would have been highly informative. Indeed, in a recent study based on voxel-based morphometry, the specific effect of posttraumatic stress disorder is described on a large sample of patients with BPD (7).

In conclusion, our results confirm that structural and functional dysfunction in subjects with BPD involves both temporolimbic and frontomedial structures and their connectivity, which, in previous studies, have been extensively related to behavioral and clinical symptoms in patients with BPD. Nevertheless, the high rates of clinical remission shown in more recent long-term follow-up studies $(69,70)$ suggest that such abnormalities may be reversible. Further research is necessary to study changes in these structural and functional abnormalities in patients before and after psychotherapeutic interventions.

\section{ACKNOWLEDGMENTS AND DISCLOSURES}

This work was supported by the Catalonian Government (Grant No. 2009SGR211), the foundation La Fundació La Marató de TV3 (Grant No. 2009-092410), and two Miguel Servet Research Contracts (Grant No. CP07/ 00048 to RS and Grant No. CP10/00596 to EP-C) from the Plan Nacional de $\mathrm{I}+\mathrm{D}+\mathrm{i}$ and cofunded by the Instituto de Salud Carlos III-Subdirección General de Evaluación y Fomento de la Investigación (Grant No. PI10/ 01071) and the European Regional Development Fund.

The authors report no biomedical financial interests or potential conflicts of interest.

\section{ARTICLE INFORMATION}

From the Fundació per a la Investigació i Docència María Angustias Giménez (RS, EJC-R, MA, EP-C), Germanes Hospitalaries, Barcelona; Centro de Investigación Biomedica en Red de Salud Mental (RS, JCP, EJC-R, MA, JS, EP-C), Barcelona; Servei de Psiquiatria i Salut Mental (DV, AS, JR), Consorci Sanitari de l'Anoia, Igualada; Departament de Psiquiatria i Medicina Legal \& Institut de Neurociències (DV), Universitat Autònoma de Barcelona, Barcelona; Department of Psychiatry (JCP, JS), Hospital de la Santa Creu i Sant Pau, Barcelona; Institut d'Investigació Biomèdica-Sant Pau (JCP, JS), Universitat Autònoma de Barcelona, Barcelona; Faculty of Psychology (JM, AR-F), University of Barcelona, Bellvitge Hospital, Barcelona; Benito Menni-Centre Assistencial en Salut Mental (SA), Sant Boi de Llobregat, Barcelona, Spain; Psychiatry and Clinical Psychology Programme (SA), Universitat Autònoma de Barcelona, Barcelona, and Hospital Sant Joan de Déu (TM), Esplugues de Llobregrat, Spain.

Address correspondence to Raymond Salvador, Ph.D., Benito MenniCentre Assistencial en Salut Mental, c/Dr. Antoni Pujadas 38, 08830 Sant Boi de Llobregat, Barcelona, Spain; E-mail: rsalvador@fidmag.com.

Received Apr 3, 2014; revised Aug 1, 2014; accepted Aug 25, 2014.

Supplementary material cited in this article is available online at http://dx.doi.org/10.1016/j.biopsych.2014.08.026.

\section{REFERENCES}

1. Grant BF, Chou SP, Goldstein RB, Huang B, Stinson FS, Saha TD, et al. (2008): Prevalence, correlates, disability, and comorbidity of DSM-IV borderline personality disorder: Results from the Wave 2 National Epidemiologic Survey on Alcohol and Related Conditions. J Clin Psychiatry 69:533-545.

2. Zanarini MC, Yong L, Frankenburg FR, Hennen J, Reich DB, Marino MF, et al. (2002): Severity of reported childhood sexual abuse and its relationship to severity of borderline psychopathology and psychosocial impairment among borderline inpatients. J Nerv Ment Dis 190: 381-387

3. New AS, Goodman M, Triebwasser J, Siever LJ (2008): Recent advances in the biological study of personality disorders. Psychiatr Clin North Am 31:441-461.

4. New AS, Hazlett EA, Buchsbaum MS, Goodman M, Mitelman SA, Newmark R, et al. (2007): Amygdala-prefrontal disconnection in borderline personality disorder. Neuropsychopharmacology 32: 1629-1640.

5. Ruocco AC, Amirthavasagam S, Zakzanis KK (2012): Amygdala and hippocampal volume reductions as candidate endophenotypes for borderline personality disorder: A meta-analysis of magnetic resonance imaging studies. Psychiatry Res 201:245-252.

6. Brunner R, Henze R, Parzer P, Kramer J, Feigl N, Lutz K, et al. (2010): Reduced prefrontal and orbitofrontal gray matter in female adolescents with borderline personality disorder: Is it disorder specific? Neuroimage 49:114-120.

7. Niedtfeld I, Schulze L, Krause-Utz A, Demirakca T, Bohus M, Schmah C (2013): Voxel-based morphometry in women with borderline personality disorder with and without comorbid posttraumatic stress disorder. PloS One 8:e65824.

8. Ridderinkhof KR, Ullsperger M, Crone EA, Nieuwenhuis S (2004): The role of the medial frontal cortex in cognitive control. Science 306 : 443-447. 
9. De La Fuente JM, Goldman S, Stanus E, Vizuete C, Morlan I, Bobes J, et al. (1997): Brain glucose metabolism in borderline personality disorder. J Psychiatr Res 31:531-541.

10. Prossin AR, Love TM, Koeppe RA, Zubieta JK, Silk KR (2010): Dysregulation of regional endogenous opioid function in borderline personality disorder. Am J Psychiatry 167:925-933.

11. Salavert J, Gasol M, Vieta E, Cervantes A, Trampal C, Gispert JD (2011): Fronto-limbic dysfunction in borderline personality disorder: A 18F-FDG positron emission tomography study. J Affect Disord 131: 260-267.

12. Ruocco AC, Amirthavasagam S, Choi-Kain LW, McMain SF (2013): Neural correlates of negative emotionality in borderline personality disorder: An activation-likelihood-estimation meta-analysis. Biol Psychiatry 73:153-160.

13. Wall PM, Messier C (2001): The hippocampal formation-orbitomedial prefrontal cortex circuit in the attentional control of active memory. Behav Brain Res 127:99-117.

14. Phillips RG, LeDoux JE (1992): Differential contribution of amygdala and hippocampus to cued and contextual fear conditioning. Behav Neurosci 106:274-285.

15. Cullen KR, Vizueta N, Thomas KM, Han GJ, Lim KO, Camchong J, et al. (2011): Amygdala functional connectivity in young women with borderline personality disorder. Brain Connect 1:61-71.

16. Niedtfeld I, Kirsch P, Schulze L, Herpertz SC, Bohus M, Schmahl C (2012): Functional connectivity of pain-mediated affect regulation in borderline personality disorder. PloS One 7:e33293.

17. Raichle ME (2011): The restless brain. Brain Connect 1:3-12.

18. Wolf RC, Sambataro F, Vasic N, Schmid M, Thomann PA, Bienentreu SD, et al. (2011): Aberrant connectivity of resting-state networks in borderline personality disorder. J Psychiatry Neurosci 36:402-411.

19. Doll A, Sorg C, Manoliu A, Woller A, Meng C, Forstl H, et al. (2013): Shifted intrinsic connectivity of central executive and salience network in borderline personality disorder. Front Hum Neurosci 7: 727.

20. Cole MW, Pathak S, Schneider W (2010): Identifying the brain's most globally connected regions. Neuroimage 49:3132-3148.

21. Buckner RL, Sepulcre J, Talukdar T, Krienen FM, Liu H, Hedden T, et al. (2009): Cortical hubs revealed by intrinsic functional connectivity: Mapping, assessment of stability, and relation to Alzheimer's disease. J Neurosci 29:1860-1873.

22. Tomasi D, Volkow ND (2010): Functional connectivity density mapping. Proc Natl Acad Sci U S A 107:9885-9890.

23. Salvador R, Sarro S, Gomar JJ, Ortiz-Gil J, Vila F, Capdevila A, et al. (2010): Overall brain connectivity maps show cortico-subcortical abnormalities in schizophrenia. Hum Brain Mapp 31:2003-2014.

24. Salvador R, Anguera M, Gomar JJ, Bullmore ET, Pomarol-Clotet E (2010): Conditional mutual information maps as descriptors of net connectivity levels in the brain. Front Neuroinform 4:115.

25. Carrasco JL, Tajima-Pozo K, Diaz-Marsa M, Casado A, Lopez-Ibor JJ, Arrazola J, et al. (2012): Microstructural white matter damage at orbitofrontal areas in borderline personality disorder. J Affect Disord 139:149-153.

26. Grant JE, Correia S, Brennan-Krohn T, Malloy PF, Laidlaw DH, Schulz SC (2007): Frontal white matter integrity in borderline personality disorder with self-injurious behavior. J Neuropsychiatry Clin Neurosci 19:383-390

27. Rusch N, Bracht T, Kreher BW, Schnell S, Glauche V, Il'yasov KA, et al. (2010): Reduced interhemispheric structural connectivity between anterior cingulate cortices in borderline personality disorder. Psychiatry Res 181:151-154.

28. Zang YF, He Y, Zhu CZ, Cao QJ, Sui MQ, Liang M, et al. (2007): Altered baseline brain activity in children with ADHD revealed by resting-state functional MRI. Brain Dev 29:83-91.

29. Yang H, Long XY, Yang Y, Yan H, Zhu CZ, Zhou XP, et al. (2007): Amplitude of low frequency fluctuation within visual areas revealed by resting-state functional MRI. Neuroimage 36:144-152.

30. Li Z, Zhu Y, Childress AR, Detre JA, Wang Z (2012): Relations between BOLD fMRI-derived resting brain activity and cerebral blood flow. Plos One 7:e44556.
31. Magri C, Schridde U, Murayama Y, Panzeri S, Logothetis NK (2012): The amplitude and timing of the BOLD signal reflects the relationship between local field potential power at different frequencies. J Neurosc 32:1395-1407.

32. Sacher J, Neumann J, Okon-Singer H, Gotowiec S, Villringer A (2013): Sexual dimorphism in the human brain: Evidence from neuroimaging. Magn Reson Imaging 31:366-375.

33. Villar Garcia M, Perez Prieto JF, Hernandez Viadel M, Renovell Farre M, Leal Cercos C, Gomez Beneyto M (1995): Preparation of a SCID-IIbased diagnostic tool for personality disorders [in Spanish]. Actas Luso Esp Neurol Psiquiatr Cienc Afines 23:178-183.

34. Barrachina J, Soler J, Campins MJ, Tejero A, Pascual JC, Alvarez E, et al. (2004): Validation of a Spanish version of the Diagnostic Interview for Bordelines-Revised (DIB-R) [in Spanish]. Actas Esp Psiquiatr 32:293-298.

35. Bohus M, Kleindienst N, Limberger MF, Stieglitz RD, Domsalla M, Chapman AL, et al. (2009): The short version of the Borderline Symptom List (BSL-23): Development and initial data on psychometric properties. Psychopathology 42:32-39.

36. Soler J, Vega D, Feliu-Soler A, Trujols J, Soto A, Elices M, et al. (2013): Validation of the Spanish version of the Borderline Symptom List, short form (BSL-23). BMC Psychiatry 13:139.

37. Pomarol-Clotet E, Canales-Rodriguez EJ, Salvador R, Sarro S, Goma JJ, Vila F, et al. (2010): Medial prefrontal cortex pathology in schizophrenia as revealed by convergent findings from multimodal imaging. Mol Psychiatry 15:823-830

38. Smith SM, Jenkinson M, Woolrich MW, Beckmann CF, Behrens TE Johansen-Berg H, et al. (2004): Advances in functional and structura MR image analysis and implementation as FSL. Neuroimage 23(suppl 1): S208-S219.

39. Smith SM, Jenkinson M, Johansen-Berg H, Rueckert D, Nichols TE, Mackay CE, et al. (2006): Tract-based spatial statistics: Voxelwise analysis of multi-subject diffusion data. Neuroimage 31:1487-1505.

40. R Development Core Team (2011): R: A Language and Environment for Statistical Computing. Vienna: R Foundation for Statistical Computing.

41. Beckmann M, Johansen-Berg H, Rushworth MF (2009): Connectivitybased parcellation of human cingulate cortex and its relation to functional specialization. J Neurosci 29:1175-1190.

42. Canales-Rodriguez EJ, Pomarol-Clotet E, Radua J, Sarro S, AlonsoLana S, Del Mar Bonnin C, et al. (2014): Structural abnormalities in bipolar euthymia: A multicontrast molecular diffusion imaging study. Biol Psychiatry 76:329-348.

43. Rossi R, Pievani M, Lorenzi M, Boccardi M, Beneduce R, Bignotti S, et al. (2013): Structural brain features of borderline personality and bipolar disorders. Psychiatry Res 213:83-91.

44. Craig MC, Catani M, Deeley Q, Latham R, Daly E, Kanaan R, et al. (2009): Altered connections on the road to psychopathy. Mol Psychiatry 14:946-953, 907.

45. Camara E, Rodriguez-Fornells A, Munte TF (2010): Microstructural brain differences predict functional hemodynamic responses in a reward processing task. J Neurosci 30:11398-11402.

46. Vega D, Soto A, Amengual JL, Ribas J, Torrubia R, Rodriguez-Fornells A, et al. (2013): Negative reward expectations in borderline personality disorder patients: Neurophysiological evidence. Biol Psychol 94 388-396.

47. Joutsa J, Saunavaara J, Parkkola R, Niemela S, Kaasinen V (2011): Extensive abnormality of brain white matter integrity in pathological gambling. Psychiatry Res 194:340-346.

48. Ungerleider LG, Gaffan D, Pelak VS (1989): Projections from inferio temporal cortex to prefrontal cortex via the uncinate fascicle in rhesus monkeys. Exp Brain Res 76:473-484.

49. Catani M, Thiebaut de Schotten M (2008): A diffusion tensor imaging tractography atlas for virtual in vivo dissections. Cortex 44:1105-1132.

50. Gaffan D, Wilson CR (2008): Medial temporal and prefrontal function: Recent behavioural disconnection studies in the macaque monkey. Cortex 44:928-935.

51. Ross ED (2008): Sensory-specific amnesia and hypoemotionality in humans and monkeys: Gateway for developing a hodology of memory. Cortex 44:1010-1022. 
52. Rusch N, Luders E, Lieb K, Zahn R, Ebert D, Thompson PM, et al. (2007): Corpus callosum abnormalities in women with borderline personality disorder and comorbid attention-deficit hyperactivity disorder. J Psychiatry Neurosci 32:417-422.

53. Zanetti MV, Soloff PH, Nicoletti MA, Hatch JP, Brambilla P, Keshavan MS, et al. (2007): MRI study of corpus callosum in patients with borderline personality disorder: A pilot study. Prog Neuropsychopharmacol Biol Psychiatry 31:1519-1525.

54. Pandya D, Seltzer B (1986): The topography of commisural fibers. In: Lepore F, Ptito M, Jasper H, editors. Two Hemispheres-One Brain: Functions of the Corpus Callosum. New York: Alan R. Liss, Inc.

55. Brambilla P, Nicoletti MA, Sassi RB, Mallinger AG, Frank E, Kupfer DJ, et al. (2003): Magnetic resonance imaging study of corpus callosum abnormalities in patients with bipolar disorder. Biol Psychiatry 54:1294-1297.

56. Brambilla P, Nicoletti M, Sassi RB, Mallinger AG, Frank E, Keshavan MS, et al. (2004): Corpus callosum signal intensity in patients with bipolar and unipolar disorder. J Neurol Neurosurg Psychiatry 75:221-225.

57. De Bellis MD, Keshavan MS, Clark DB, Casey BJ, Giedd JN, Boring AM, et al. (1999): A.E. Bennett Research Award. Developmental traumatology. Part II: Brain development. Biol Psychiatry 45:1271-1284.

58. Teicher MH, Dumont NL, Ito Y, Vaituzis C, Giedd JN, Andersen SL (2004): Childhood neglect is associated with reduced corpus callosum area. Biol Psychiatry 56:80-85.

59. Hoptman MJ, Zuo XN, Butler PD, Javitt DC, D’Angelo D, Mauro CJ, et al. (2010): Amplitude of low-frequency oscillations in schizophrenia: A resting state fMRI study. Schizophr Res 117:13-20.

60. Yu R, Chien YL, Wang HL, Liu CM, Liu CC, Hwang TJ, et al. (2014): Frequency-specific alternations in the amplitude of low-frequency fluctuations in schizophrenia. Hum Brain Mapp 35:627-637.

61. Guo WB, Liu F, Xue ZM, Xu XJ, Wu RR, Ma CQ, et al. (2012): Alterations of the amplitude of low-frequency fluctuations in treatment-resistant and treatment-response depression: A resting-state fMRI study. Prog Neuropsychopharmacol Biol Psychiatry 37:153-160.
62. Hou J, Wu W, Lin Y, Wang J, Zhou D, Guo J, et al. (2012): Localization of cerebral functional deficits in patients with obsessive-compulsive disorder: A resting-state fMRI study. J Affect Disord 138:313-321.

63. Raichle ME, MacLeod AM, Snyder AZ, Powers WJ, Gusnard DA, Shulman GL (2001): A default mode of brain function. Proc Natl Acad Sci U S A 98:676-682.

64. Northoff G, Bermpohl F (2004): Cortical midline structures and the self. Trends Cogn Sci 8:102-107.

65. Vago DR, Silbersweig DA (2012): Self-awareness, self-regulation, and self-transcendence (S-ART): A framework for understanding the neurobiological mechanisms of mindfulness. Front Hum Neurosci 6: 296.

66. Holtmann J, Herbort MC, Wustenberg T, Soch J, Richter S, Walter H, et al. (2013): Trait anxiety modulates fronto-limbic processing of emotional interference in borderline personality disorder. Front Hum Neurosci 7:54

67. Jacob GA, Zvonik K, Kamphausen S, Sebastian A, Maier S, Philipsen A, et al. (2013): Emotional modulation of motor response inhibition in women with borderline personality disorder: An fMRI study. J Psychiatry Neurosci 38:164-172.

68. Silbersweig D, Clarkin JF, Goldstein M, Kernberg OF, Tuescher O, Levy KN, et al. (2007): Failure of frontolimbic inhibitory function in the context of negative emotion in borderline personality disorder. Am J Psychiatry 164:1832-1841.

69. Gunderson JG, Stout RL, McGlashan TH, Shea MT, Morey LC, Grilo CM, et al. (2011): Ten-year course of borderline personality disorder: psychopathology and function from the Collaborative Longitudinal Personality Disorders study. Arch Gen Psychiatry 68:827-837.

70. Zanarini MC, Frankenburg FR, Reich DB, Fitzmaurice G (2012): Attainment and stability of sustained symptomatic remission and recovery among patients with borderline personality disorder and axis II comparison subjects: A 16-year prospective follow-up study. Am J Psychiatry 169:476-483. 